\title{
SoC Estimation in Li-ion Batteries Exploiting High-Frequency Model Properties
}

\author{
Pablo García, Ángel Navarro-Rodríguez, Sarah Saeed and Jorge García \\ University of Oviedo. Dept.of Elec., Computer \& System Engineering \\ Gijón, 33204, Spain \\ e-mail: garciafpablo@uniovi.es, navarroangel@uniovi.es, hazkialsarah.uo@uniovi.es, garciajorge@uniovi.es
}

\begin{abstract}
This paper explores the use of the high-frequency voltages and currents at battery terminals for the estimation of the State of Charge (SoC). The proposed approach will analyze the viability of using both the switching harmonics injected by the power converter used for the interface of the battery as well as an additional high-frequency signal excitation. Over the high-frequency model of the battery, the variation of the model parameters with respect to the fundamental current and SoC value is analyzed. Using the variation of one of the model inductive terms, a SoC estimation procedure is presented. The proposed methodology can be later extended to analyze the influence of other important values, as the State of Health (SoH) and cell temperature.
\end{abstract}

\section{INTRODUCTION}

Use of Li-ion batteries have shown a significant boost in different energy storage applications, from electrical transportation to stationary grid support [1], [2]. According to its cell geometry and chemistry, the properties of the energy storage system (ESS) could be more devoted to powerdemanding or energy-demanding applications [3]. Regarding the cell geometry, the more typical configurations are cylindrical and prismatic. About chemistries, there are a number of alternatives. Some of the most popular are Lithium Nickel Manganese Cobalt Oxide (NMC), Lithium Iron Phosphate (LFP) and Lithium Titanate (LTO). A comparative analysis for three different configurations is shown in Table I.

TABLE I

Li-ION CELl CONFIGURATIONS

\begin{tabular}{lcccc}
\hline Battery & $\begin{array}{c}P_{r} \\
(\mathrm{~W})\end{array}$ & $\begin{array}{c}E_{r} \\
(\mathrm{Wh})\end{array}$ & $C_{c h} / C_{\text {disch }}$ & cycles \\
\hline NMC & 481 & 161 & $2 / 3$ & 3800 \\
LFP (cylindrical) & 396 & 66 & $4 / 6$ & 7300 \\
LTO & 1144 & 143 & $4 / 8$ & 15100 \\
LFP (prismatic) & 1147 & 573 & $1 / 2$ & 5000 \\
\hline
\end{tabular}

The present work has been partially supported by the predoctoral grants program Severo Ochoa for the formation in research and university teaching of Principado de Asturias PCTI-FICYT under the grants ID BP14-135 and BP16133. This work has been partially supported by the government of Principality of Asturias, under IDEPA grant 2017 Thyssen SV-PA-17-RIS3-3. This work also was supported in part by the Research, Technological Development and Innovation Program Oriented to the Society Challenges of the Spanish Ministry of Economy and Competitiveness under grant ENE2016-77919-R and by the European Union through ERFD Structural Funds (FEDER).
Depending on the ESS specifications, the battery is built by arranging internal cells in series, the so call strings, and the strings in parallel connections to get a module. The modules can also be interconnected in series or parallel to obtain the final battery-pack. Coupled together to each module, there is a dedicated control system, namely battery management system (BMS), which is responsible of monitoring the different parameters needed to guarantee the safe operation of the system [4]. Among those, the most important are the internal cell voltages, temperatures, SoC and $\mathrm{SoH}$ [5]. In order to accomplish the estimation of the aforementioned variables, the BMS often use voltage, current and temperature sensors. Depending on the monitored variable, the critical values to estimate are: 1) minimum and maximum cell voltage, 2) minimum and maximum cell temperature, 3) internal impedance, 4) $\mathrm{SoH}$ and 5) SoC. From the previous defined variables, 1) and 2) are directly obtained from the sensors readings, whereas the last three need from estimation algorithms.

As discussed above, estimation of critical operation and safe related parameters and values in battery-based ESS needs for bespoke algorithms. The battery SoH can be determined either from the value of the equivalent internal cell impedance or from the open circuit voltage (OCV) [6]. Considering the fact that the ESS will often have a dedicated current sensor for protection purposes, the internal impedance can be determined combining together the information from the voltage and current sensors. The procedure, even if quite tedious, can be easily done in offline tests, being a popular method for the characterization of the impedance the electrochemical impedance spectroscopy [7]. However, the value of the impedance is affected by the operation point of the battery, making the online implementation more difficult [6].

Estimation of battery SoC is undoubtedly the most demanded feature from battery manufactures. The literature in this direction is vast and different alternatives have been proposed [8]. The different approaches can be separated into model-based, signal-based or hybrid model-signal techniques. The first group depends on an accurate model of the battery. This model could be implemented in different domains: electrochemical and electrical equivalent [9]. The first approach goes down to the physical description of the cell and due to its detailed view is more accurate, even if it needs for solving non- 
linear equations and adjustment of various parameters [10]. The second approach is simpler and is the alternative usually taken. It is based on the equivalent electrical circuit describing the battery internal behavior and is basically an ideal voltage source representing the open-circuit voltage (OCV) series connected to a resistance the electric losses and a number of RC networks. Using this approach, SoC is estimated by measuring the terminal voltage and current and determining the OCV, which is a non-linear function of the SoC [11]. Signal-based methods are the simplest ones and estimate the SoC based on the circulating current. Considering the rated capacity of the battery, and calculating the time-integration of the current, the actual charge can be easily determined. However, the method is greatly affected by offsets in the current sensor [12]. Hybrid methods combine together model and signal methods by estimation techniques, ranging from RLS, Kalman filters or neural networks approaches [13]-[15].

This paper proposes the use of a hybrid technique based on the high-frequency model of the battery [16], [17], that is suitable for the calculation of the SoC and that is barely affected by the operating point of the battery. The underpinning idea is to use the harmonics delivered by the power converter needed for the battery interface. Those harmonics can be caused due to the natural switching of the converter or because of an additional high-frequency signal injected for estimation purposes. At first sight, the first alternative is preferred because that makes the proposed method suitable to be implemented without any modification in the power converter itself and avoid any extra losses due to the additional signal injection. Still, the two approaches are explored in order to make a comparative sensitivity analysis. The proposed method can be also used for $\mathrm{SoH}$ and internal temperature estimation.

This paper is organized as follows. Section II presents the high-frequency model used for the cell and module modelling as well as the estimation procedure used for obtaining the parameters. Section III explains the two evaluated highfrequency signal injection alternatives. Section IV presents the experimental results for the SoC estimation based on the highfrequency model. Finally, V enumerates the conclusions and contributions of the paper.

\section{HIGH-FREQUENCY MODEL}

In order to derive the battery-pack high-frequency model, the battery can be modeled at cell, module or even at batterypack level. A cell-level model has the advantage of capturing the internal high-frequency behavior of the individual cells, which can be reused for estimating the high-frequency behavior for different configurations of series/parallel cells. However, it does not considers any additional impedance resulting for the cells integration into a module.

By the other hand, module-level modelling makes the estimation to be affected by the additional impedance resulting from the cells soldering as well the cabling. If both cellmodel and module-level model are obtained, the effect of this additional elements over the estimation procedure can be easily quantified. Additionally, this triggers the opportunity of implementing the estimation techniques by only using the voltage and current at module-level, which are usually available or easily measured. Under this scheme, the proposed estimation technique can be implemented either at modulelevel control system or at the power converter itself. In this paper, the module-level impedance is used.

\section{A. High-frequency model estimation.}

The parameters of the battery module have been estimated by a vector fitting method [17], [18]. On obtaining the proposed high frequency model, it has to be mentioned that the impedance spectrum of a battery is dependent on the actual SoC, the temperature and cycling [6], [19], [20]. The $\mathrm{SoC}$ effect will be later used, during the presentation of the experimental results, as an estimator. As presented in [21], the cell-level impedance-based high frequency model shown in Fig. 1, consists of two resistors and two inductors. The parameters of this model can be derived by approximating the frequency response of the cells for different SoC with the expression shown in (1), where $s$ is the Laplace variable.

$$
f(s) \approx \sum_{m=1}^{N} \frac{c}{s-a}+d+s e
$$

The relationship between the coefficients shown on (1) and the

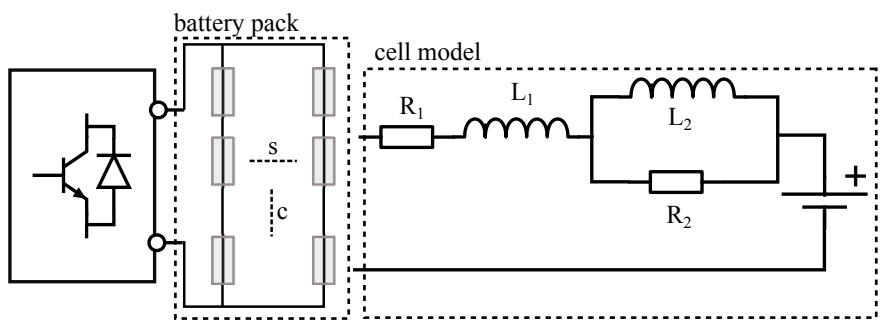

Fig. 1. Battery high frequency model representation as seen from the converter terminals.

high-frequency model parameters have been done as follows. Using the equivalent high-frequency model shown in Fig. 1, the cell high frequency impedance is given by (2).

$$
Z_{\text {cell }}(s)=R_{1}+s L_{1}+\frac{L_{2} s R_{2}}{L_{2} s+R_{2}}
$$

By comparing the polynomial coefficients of (1) and (2), the equivalences in (3) and can be obtained.

$$
R_{1}=\frac{d \cdot a-c}{a} ; \quad R_{2}=\frac{c}{a} ; \quad L_{1}=e ; \quad L_{2}=-\frac{c}{a^{2}}
$$

The above presented model is valid both for describing the cell-level behavior, but also the module and battery-level models. For that, the electrical connection among the cells has to be specified and, considering the battery-pack be formed by a number of identical series-connected modules and each of the modules by parallel strings built by series connected cells, an expression on the form of (4) can be obtained. In (4), $m$ refers to the number of series-connected modules for building the battery-pack, $s$ the number of parallel strings per module and $c$ the number of series cells per string. It is important to 
TABLE II

BATTERY MODULE PARAMETERS.

\begin{tabular}{ll}
\hline Parameter & Value \\
\hline rated voltage & $48 \mathrm{~V}$ \\
rated capacity & $4.8 \mathrm{kWh}$ \\
rated current (1C) & $100 \mathrm{~A}$ \\
series cells & 15 \\
parallel strings & 1 \\
\hline
\end{tabular}
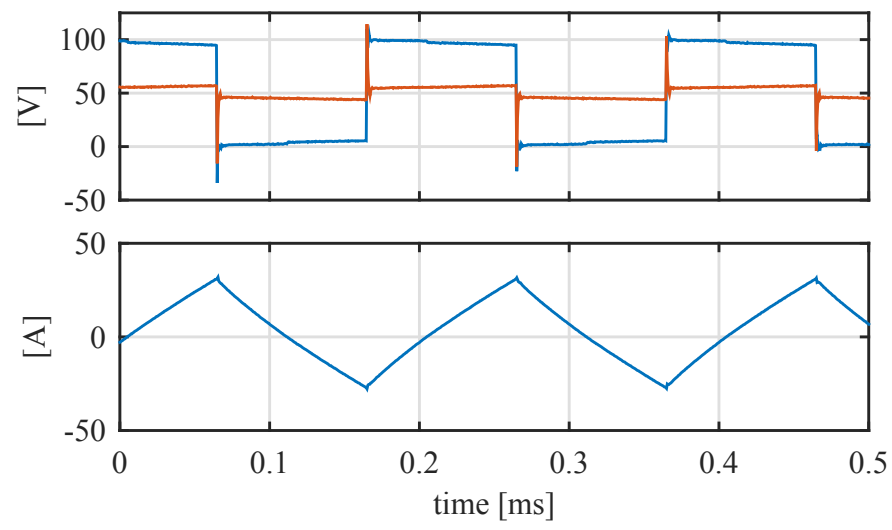

Fig. 2. Measuring of module-level high-frequency impedance. On top, converter-terminal voltage (blue) and battery-terminal voltage (red). On bottom, circulating current.

remark that, as said before, this model does not capture any additional impedance coming from the cells connection.

$$
Z_{e q}=\sum_{m=1}^{m=M}\left(\frac{1}{\sum_{s=1}^{s=S}\left(\frac{1}{\sum_{c=1}^{c=C} Z_{c e l l}(s)}\right)}\right)
$$

\section{B. Module high-frequency model.}

Using the proposed high frequency model shown in (2), the high frequency response at module level has been measured. The used module is a ROOK 48V100Ah module from Cegasa Portable. The module parameters are listed in Table II. A three-leg interleaved dc/dc power converter has been used for the interface with the battery. A periodic square-wave voltage disturbance has been added over a dc voltage equal to the measured battery open circuit voltage, $v_{o c v}$, and the voltage and current at the module terminals have been measured using a scope with a sample time of $0.5 \mu$ s. Isolated voltage probes (Yokogawa $700924100 \mathrm{MHz}$ bandwidth, $100: 1,2 \%$ gain accuracy) were used for the voltage measurements and current probes (Yokogawa 701930 DC-10MHz, 300A-Peak, $1 \%$ accuracy) for the currents.

Fig. 2 shows the applied voltage in the converter terminals, the voltage at the battery terminals $\left(V_{d c}^{\text {ess }}\right)$ and the resulting current $\left(I_{d c}^{e s s}\right)$. As it can be seen, a significant amount of voltage drops in the converter interface filter. This makes needed to carry out a detailed analysis for the selection of the high-frequency signal, in terms of frequency and magnitude.

Using the recorded waveforms, as shown in Fig. 2, the module-level impedance has been measured in frequency
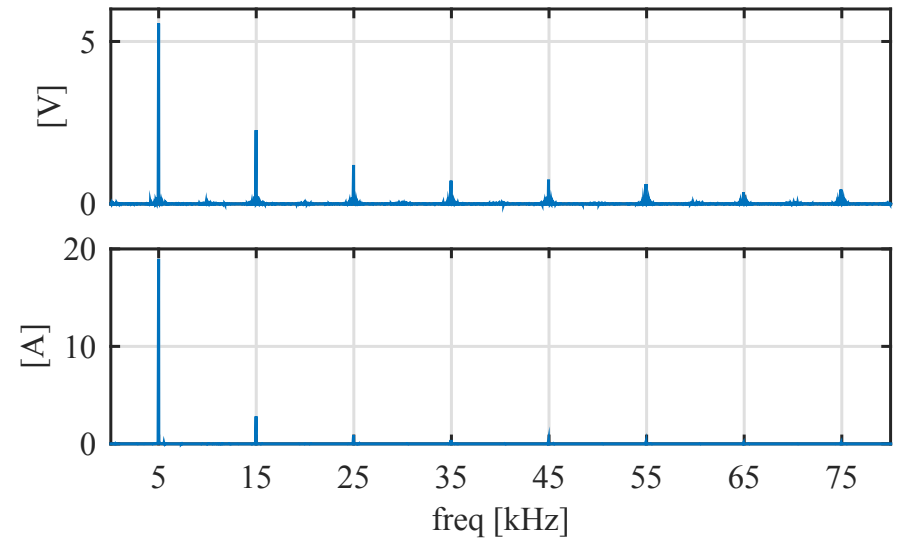

Fig. 3. Measuring of module-level high-frequency impedance in frequency domain. On top, battery-terminal voltage. On bottom, circulating current.
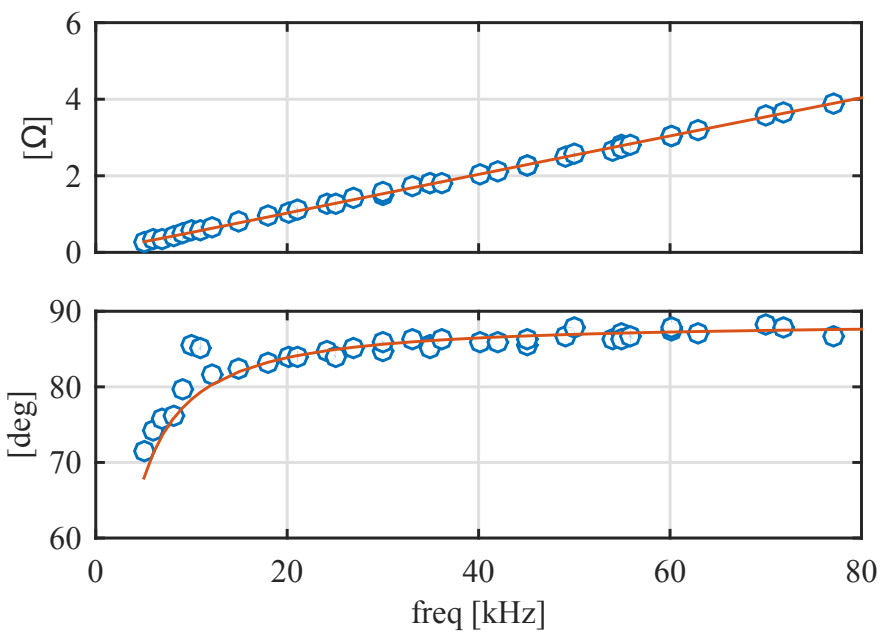

Fig. 4. Comparison between the identified module high frequency model (blue) for a $60 \% \mathrm{SoC}$ and the measured module response (red).

domain using the expression $Z_{\text {ess }}=\frac{f f t\left(V_{d c}^{\text {ess }}\right)}{f f t\left(I_{d c}^{\text {ess }}\right)}$. In order to have a wide-frequency estimation, several experiments varying the frequency of the square-wave voltage have been carried out. The input frequency was varied within the range $[5,6,7,8,9,10,11,12,20] \mathrm{kHz}$. The impedance has been measured not only at the injection frequency, but at the resulting harmonics because of the square waveform. The frequencydomain battery voltage and current are shown in Fig. 3 .

The resulting measured impedance, $Z_{\text {ess }}$, has been used as an input to the vector-fitting method explained before, which output are the parameters for the high-frequency model shown in (4). The results results are shown in Fig. 4 for a $60 \%$ SoC. Similar results are obtained for all the analyzed SoC. Clearly the proposed model is a good approximation at the studied switching frequency harmonics region $(10-80 \mathrm{kHz})$.

Using the same procedure explained before for all the SoC levels, allows to obtain the estimated model parameters, $\left[R_{1}, R_{2}, L_{1}, L_{2}\right]$ in function of the $\mathrm{SoC}$. The results are shown in Fig. 5. As clearly visible, there is a variation with respect to the SoC, which is particularly interesting for the $L_{1}$ value due 
to its linearity. This will be later used for the SoC estimation. It is also worth noting the negative value for $R_{2}$ and $L_{2}$ for the $100 \%$ SoC. This could be caused by an additional degree of freedom not considered in the model for high SoC values, in which the non-linearities are more noticeable.

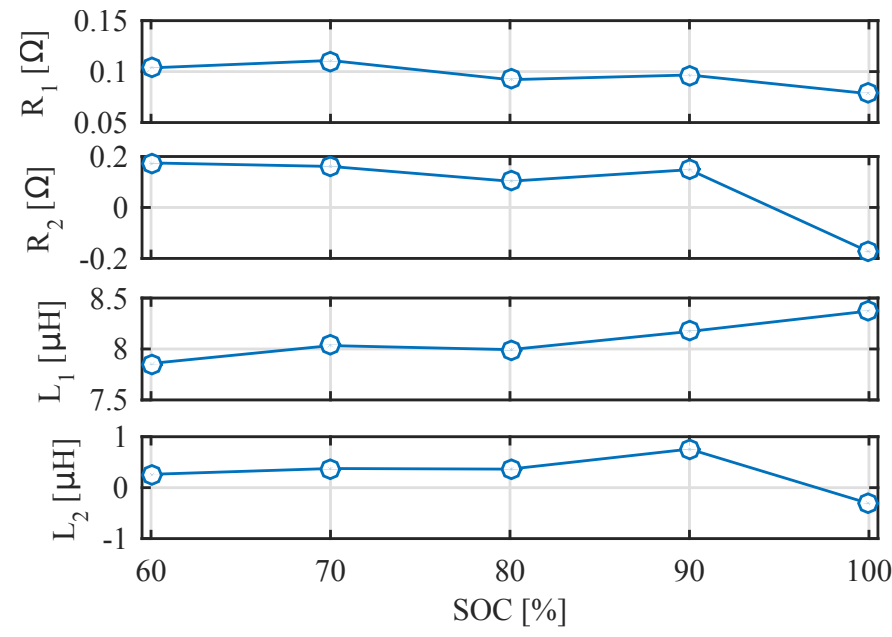

Fig. 5. Experimental results. Value of high-frequency model parameters depending on the SoC.

\section{Signal InJECTION ALTERNATIVES}

High frequency signal excitation can be delivered in different ways by the coupling dc/dc power converter. Considering the power converter is coupled to the battery-pack by a filter, it is needed to consider the effect of the filter impedance over the signal excitation. As shown before in Fig. 2, the converter filter impedance greatly attenuates the high-frequency signal and thus the proposed high-frequency-based SoC estimation. Being the filter impedance also dependent on the converter design, it is needed to understand the converter topolgy dependent parameters that can potentially affect the estimation. In this paper, a three-leg interleaved non-isolated bidirectional boost converter is considered, as shown in Fig. 6. The relevant parameters for the power converter are listed in Table III. The interleaving design is considered for two different reasons: 1) power sharing among the different legs and 2) reduction of switching harmonics by carrier phase-shifting. Obviously, this second effect could affect the proposed method. Regarding the control system, the power converter is operated in currentcontrol mode, even if the proposed method is suitable in the case voltage-mode operation is required. The interaction of the control system with the high-frequency signal injection needs also to be considered. In the case of an additional signal at frequencies well below the switching frequency, the bandwidth of the current controller can reach the frequency of interest and its reaction can also deteriorate the signal information. In the following sections, these effects are analyzed for the two considered high-frequency-based SoC estimation: 1) additional signal excitation and 2) switching harmonics.

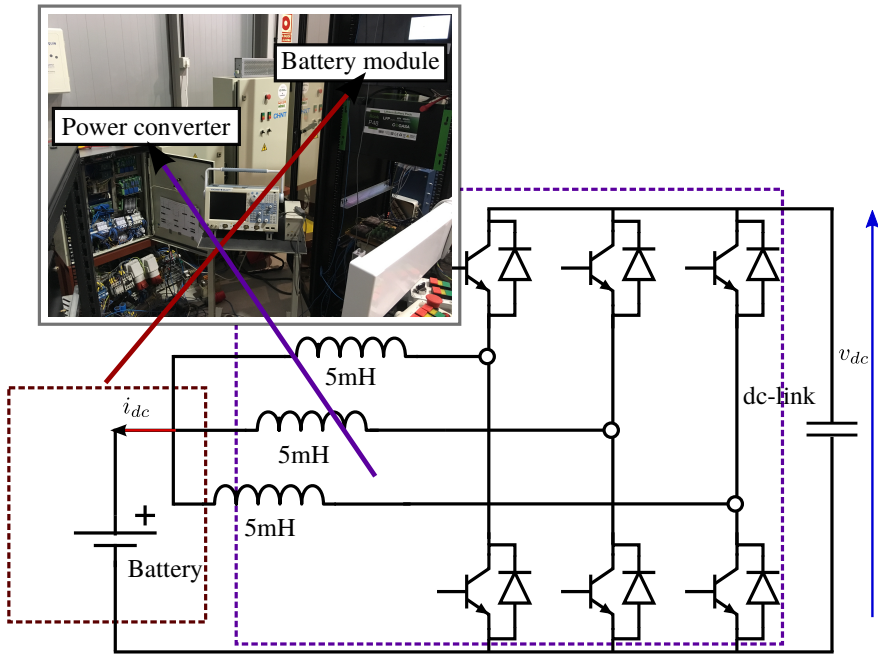

Fig. 6. Schematic for the battery interface power converter. Three-phase bidirectional interleaved boost converter is used.

TABLE III

POWER CONVERTER PARAMETERS.

\begin{tabular}{ll}
\hline Parameter & Value \\
\hline switching frequency & $10-20 \mathrm{kHz}$ \\
dc-link voltage & $650 \mathrm{~V}$ \\
filter & $5 \mathrm{mH}$ \\
current control bandwidth & $500 \mathrm{~Hz}$ \\
\hline
\end{tabular}

\section{A. Additional high-frequency signal excitation}

In this case, the high-frequency signal is injected in all or one of the interleaving legs. Two different kind of signals have been tested: squared and sinusoidal at different frequencies: $[500,1000,2000] \mathrm{Hz}$. As being told, the effect of the series connected filter at the output of the $\mathrm{dc} / \mathrm{dc}$ converter has to be accounted for, being the selection of the signal frequency and magnitude the critical parameters. Considering that the inductor filter and the battery are series connected, the applied input voltage excitation will be divided between the filter and the battery in a relation inverse proportional to their impedance at the injection frequency. As shown in section II, the battery impedance model at high frequencies becomes inductive. That would mean increasing the signal frequency will also increase the reflected voltage over the battery terminals, and thus the signal to noise ratio when the impedance is calculated. Also, increased injection frequencies will reduce the distortion by the current regulator. Being the signal injected as a voltage command added to the output of the current controller, makes the controller to see the signal as a disturbance that will try to compensate. As higher the frequency is, the lower the impact will considering the bandwidth of the controller. By the other hand, higher frequencies will reduce the circulating current as a result of the overall inductive behavior of the battery and the converter filter. Moreover, the highest the injection frequency is, the highest the derived harmonics due to the reduced number of points sampled by the modulator. 

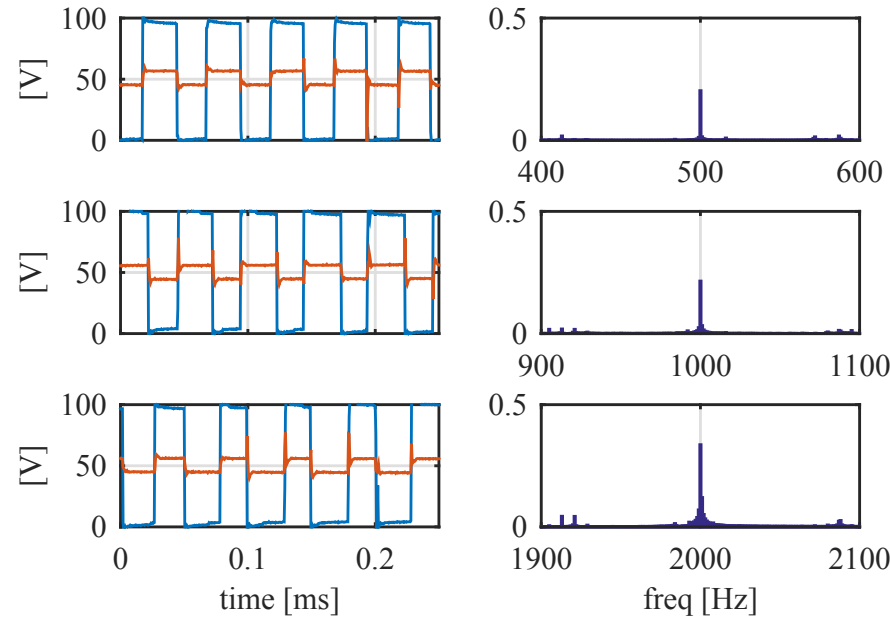

Fig. 7. Experimental results. Applied voltage modulation for the sinusoidal signal injection. Left column shows in blue the applied modulation voltage and in red the voltage at the battery terminals. On the right column the spectrum centered at the different injection frequencies is shown. Each row corresponds to a different signal injection frequency. From top to bottom: $500 \mathrm{~Hz}, 1000$ $\mathrm{Hz}$ and $2000 \mathrm{~Hz}$.
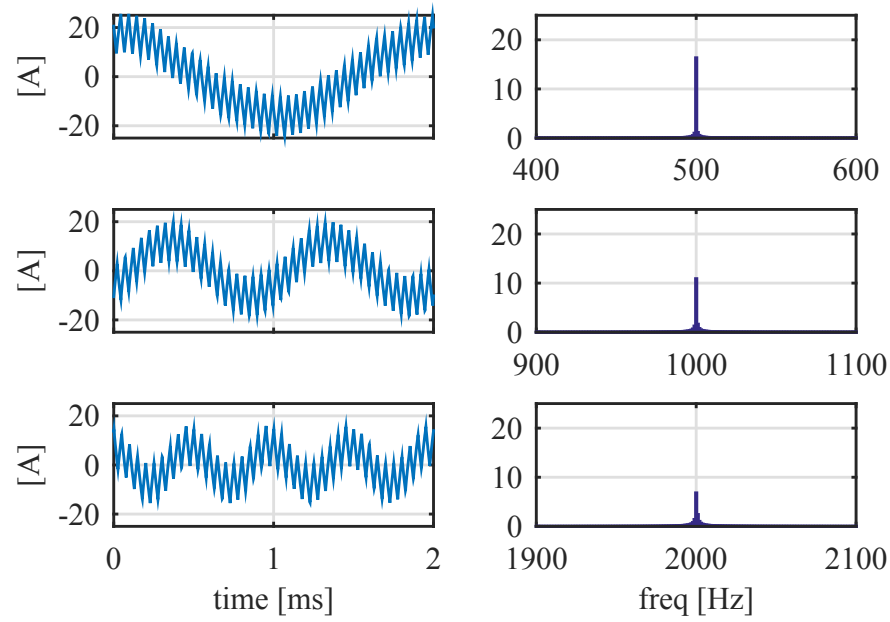

Fig. 8. Experimental results. Resulting current for the sinuoidal signal injection. Left column shows the current waveform in time-domain. On the right column the spectrum centered at the different injection frequencies is shown. Each row corresponds to a different signal injection frequency. From top to bottom: $500 \mathrm{~Hz}, 1000 \mathrm{~Hz}$ and $2000 \mathrm{~Hz}$.

Considering that the highest possible injection frequency is half the switching frequency, according to Nyquist principle, the maximum frequency for our configuration is $10 \mathrm{kHz}$ (half the switching frequency).

The experimental results regarding the signal injection at the three selected frequencies using a sinusoidal modulation, are shown in Fig. 7 and 8 for the voltage and the current respectively. As it is clearly visible, the inductive behavior of the filter and the battery makes the circulating current to be reduced with the frequency of the injection signal.

Considering the high-frequency behavior of the battery model, the selected frequency for the injection signal has been set to the highest one tested, i.e. $2000 \mathrm{~Hz}$.
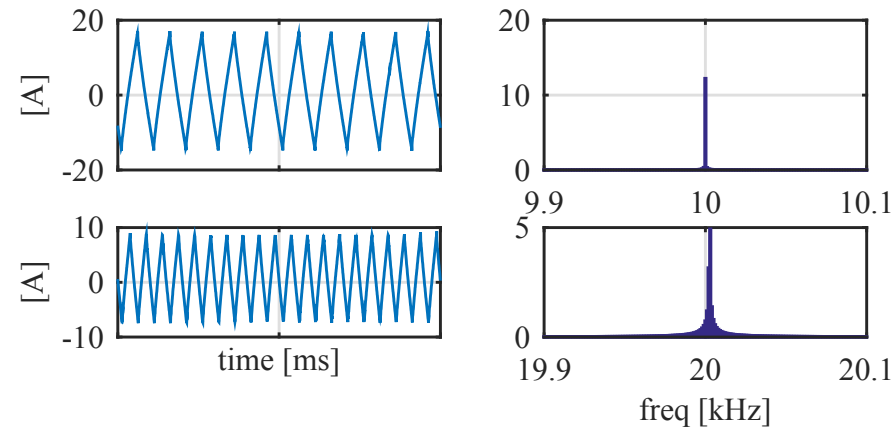

Fig. 9. Experimental results. Resulting current for the switching harmonics. Left column shows the current waveform in time-domain. On the right column the spectrum centered at the different switching frequencies is shown. Top and bottom row correspond to 10 and $20 \mathrm{kHz}$ switching frequencies.

\section{B. Switching harmonics}

The commutation of the dc/dc converter will induce switching harmonics over the connected loads, i.e, the battery. Those additional harmonics are a potential excitation signal to measure the high-frequency impedance and thus estimating battery parameters and SoC. The resulting current waveform and the corresponding spectrum for two different switching frequencies, 10 and $20 \mathrm{kHz}$ are shown in Fig. 9

Considering a sine-triangle PWM modulation, odd harmonics of the switching frequency will be induced. Tracking of the switching harmonics has some advantages over the additional signal injection: 1) Reduced losses, 2) more headroom for the delivery of the fundamental current command, 3) ability of tracking the high-frequency impedance without any modification in the power converter control. For those reasons, this is the preferred method for the excitation signal. Regarding the important parameters affecting the resolution of the method, is needed to consider the impact of the dc-link voltage value, switching frequency and modulation strategy. The dc-link value will directly determine the maximum magnitude of the main switching harmonic. Considering the switches are fired in complementary mode, the applied voltage at the output of the power converter will be $\left[V_{d c}, 0\right]$. Regarding the switching frequency, as higher it is, the lower will be the resulting current, thus making the measurement more difficult. However, as previously discussed, the difference between the impedance values at different SoC levels is higher, thus making easier the estimation. Considering the modulation strategy, any method aiming to reduce the harmonic losses will negatively affect the estimation. In our case, the use of the interleaved converter mitigates the magnitude of the switching harmonics and its impact shall be quantified.

\section{Selection of high-frequency excitation}

As previously discussed, selection of the high-frequency signal excitation requires to quantify the effect of the converter (filter, modulation strategy) as well as the sensitivity of the high-frequency model to $\mathrm{SoC}$ variations.

Regarding the effect of the converter filter, it is interesting to calculate the ratio of the reflected voltage at the battery 

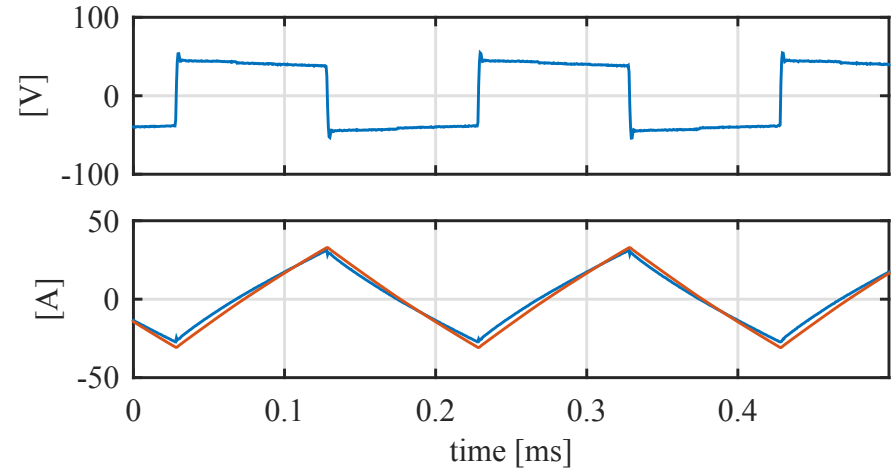

Fig. 10. Experimental results. Converter filter response. Top, voltage drop at the filter. Bottom, circulating current (blue) and predicted by the filter model (red).

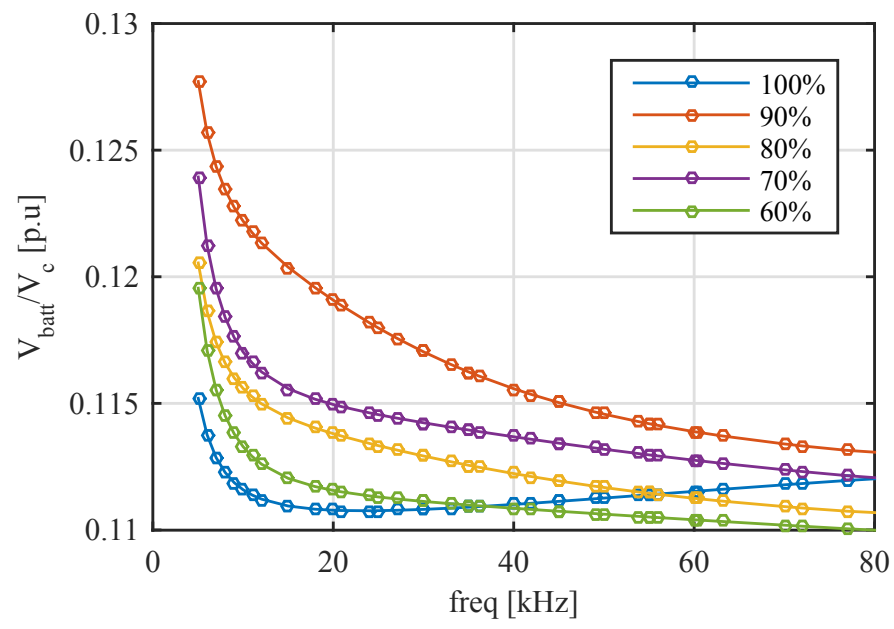

Fig. 11. Reflected battery terminal voltage at different injection frequencies. Values for different SoC, as shown in the legend, are represented.

terminals with respect to the applied input voltage as a function of the frequency and with the battery having different SoC values. The converter filter equivalent impedance was approximated by a series RL network, using the voltage drop in the filter and the output current according to the expression $V_{\text {filter }}=R_{f} \cdot i_{d} c+L_{f} \frac{d i_{d c}}{d t}$. The voltage drop as well as the measured and the approximated currents are shown in Fig. 10. The obtained parameters are $L_{f}=0.065 \mathrm{mH}$ and $R_{f}=0.05$ $\Omega$. The results are shown in Fig. 11. As it can be seen, the voltage drop at the converter filter inductance is close to $90 \%$ of the overall voltage, significantly affecting the amount of useful signal arriving to the battery terminals. Also, it can be seen the slight variations in the voltage for the different SoC, which is in agreement with the idea supporting the paper about the dependency of the high-frequency model with the SoC.

Considering the sensitivity analysis, it is important to determine the variation of the impedance module at the different frequencies of interest with respect to the SoC. In Fig. 12 the frequency-domain evaluation of the proposed model is shown. The two different regions of interest, at the sinusoidal harmonic injection and at the main switching harmonic are

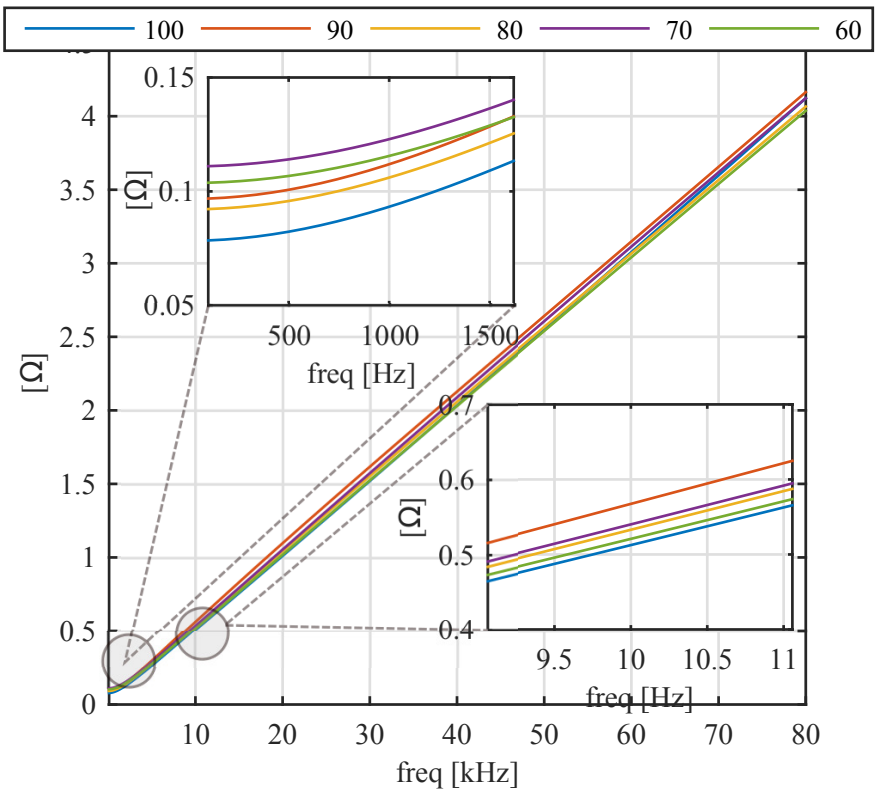

Fig. 12. Variation of the high-frequency model with respect to the SoC. Two different regions are represented in the zoomed graphs. On top, the region in the vicinity of the additional signal injection. On bottom, the first switching harmonic.

shown in detail. Some conclusions can be grabbed from the representation: 1) The lower frequencies have a variation which is non-linear. Even if that behavior is not critical once selected the injection frequency, it indicates that the resistance terms are having an important contribution. As discussed before, the term which shows a larger variation with respect to the SoC is $L_{1}$, that makes the injection in that region to be less robust. 2) The variation in the module impedance from the minimum SoC to the larger SoC is also larger at higher frequencies. For these two reasons, the switching harmonic impedance is a better candidate to be used for the estimation. 3) Selecting a higher switching frequency $(20 \mathrm{kHz})$ makes the variation in the impedance with respect to the SoC to be higher. On the contrary, the value of the resulting current is smaller due to the inductive behavior.

\section{EXPERIMENTAL RESULTS}

The proposed method has been initially evaluated by experimental results. The carried out test show really promising results later to be extended during the full paper development.

For the experimentation, a ROOK 48V100Ah module from Cegasa Portable Energy, which parameters are listed in Table II has been used. The module is shown in Fig. 6. During the carried out experiments, the SoC estimation given by the BMS as well as the minimum and maximum cell voltages have been saved with a resolution of $1 \mathrm{~s}$.

As told before, two injection methods have been used: sinusoidal high-frequency signal and the first switching harmonic. For the final experiments, the selected frequencies were 2500 $\mathrm{Hz}$ for the sinusoidal signal injection and $10 \mathrm{kHz}$ as the switching frequency. The resulting voltages and current are 

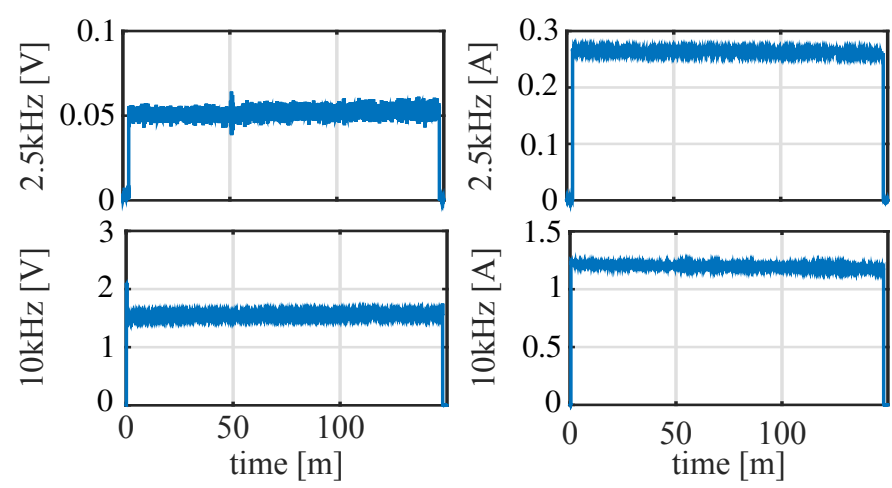

Fig. 13. Experimental results. Top row, high-frequency sinusoidal injection. Bottom row, $1^{\text {st }}$ switching harmonic injection. Left column, voltage. Right column, current.
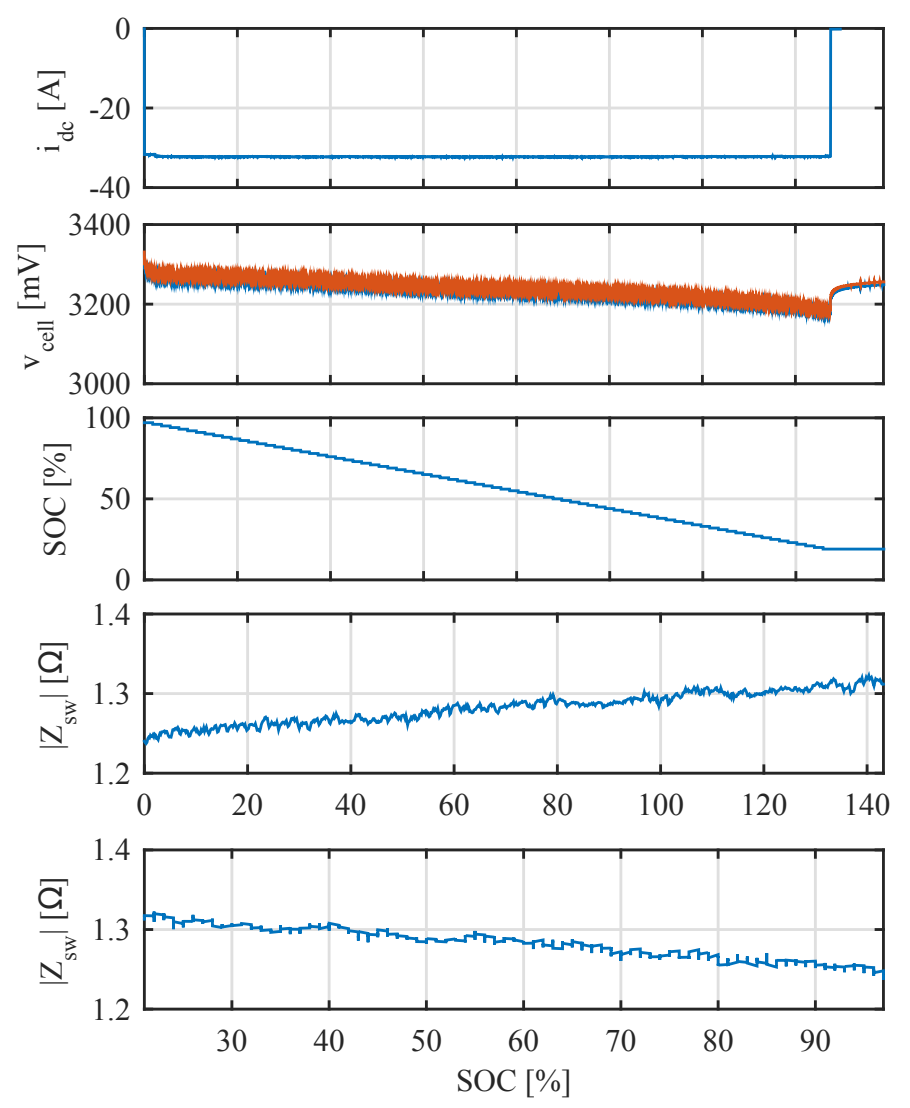

Fig. 14. Experimental results. Variation of switching impedance during discharge. From top to bottom: a) battery current, b) $\mathrm{min} / \mathrm{max}$ cell voltages, c) BMS estimated SoC, d) switching frequency impedance, e) switching frequency impedance vs. SoC.

shown in Fig. 13. Even if both signals can be used for the impedance calculation, the voltage at $2500 \mathrm{~Hz}$ is too low due to the lower impedance of the battery at that frequency. For that reason, at this paper, only the SoC estimation using the fundamental switching harmonic are shown.

Fig. 14 shows the evolution of the tracked variables during a constant current discharge at $\mathrm{C} / 3(30 \mathrm{~A})$ over the rated current of the battery. As expected, due to the constant current, the
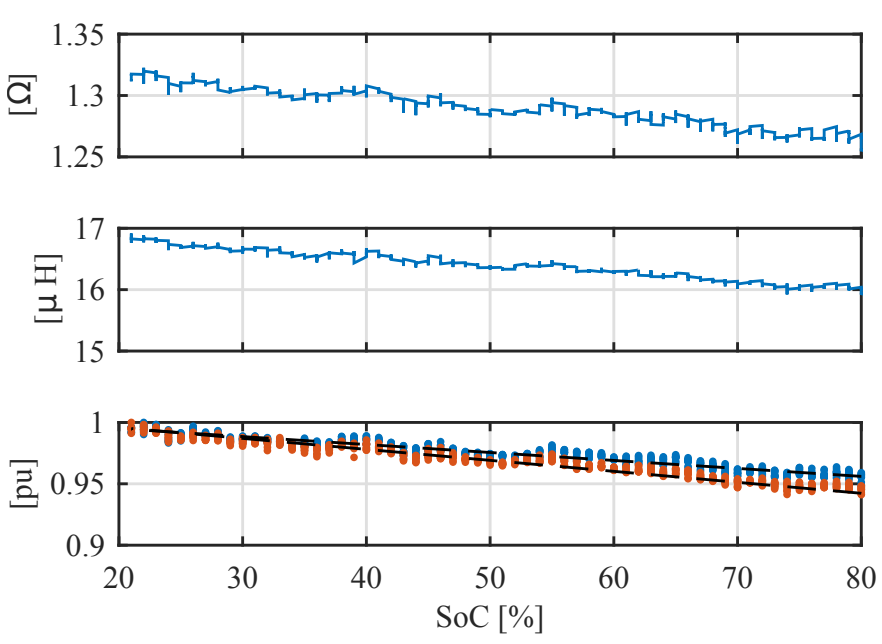

Fig. 15. Experimental results. Sensitivity analysis for the SoC estimation using the impedance or the inductive term. From top to bottom, a) impedance variation with respect to $\mathrm{SoC}, \mathrm{b}$ ) inductance variation and c) normalized impedance (blue) and inductance (red).

SoC given by the BMS decreases following a linear shape. In the last two rows of the figure, the value of the module of the impedance at the switching frequency $(10 \mathrm{kHz})$, as well as the change of that impedance with respect to the BMS SoC, are represented. Some interesting conclusions can be drawn for this experiment: 1) the switching harmonic impedance increases following a linear evolution while the $\mathrm{SoC}$ is decreasing. This linear evolution dependence with the $\mathrm{SoC}$ is also clearly visible at the last plot of the figure. Analyzing the change in the high-frequency impedance, the module variation is about $10 \%$ for a SoC variation from $100 \%$ to $20 \%$. This gives a good resolution for using the impedance as a $\mathrm{SoC}$ estimator.

As discussed when analyzing the high-frequency model, the inductive term $L_{1}$ had a higher sensitivity to SoC variations. In Fig. 15, a comparison between the sensitivity of the overall impedance and the inductive term is shown. Clearly, the inductive term is a better candidate for the $\mathrm{SoC}$ estimation. This can be quantified by approximating the two curves by a linear regression. For the case of the normalized impedance with respect to the Soc, the slope is -0.0006 , whereas for the case of using the normalized inductance, the slope increases up to -0.0009 . For both cases units are [pu/\%].

Fig. 16 shows the variation of the switching harmonic impedance when charge/discharge current pulses are applied during $1 \mathrm{~m}$. During the experiment, the estimated SoC by the BMS remained stable at $77 \%$. As it can be seen, the value of the impedance remains is also kept with small variation with respect to the initial value. Even with the limited experimental results presented at this paper, the use of the switching harmonic impedance seems promising for SoC estimation.

\section{CONClusion}

This paper has shown the use of the battery high-frequency properties for estimating the SoC. The method has been 

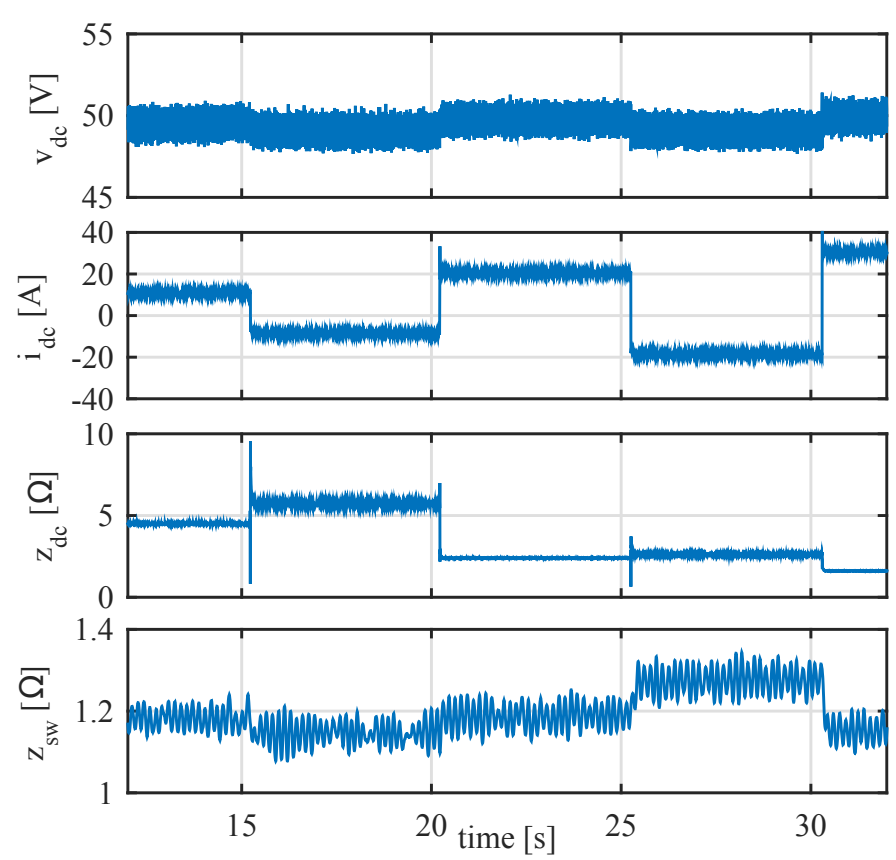

Fig. 16. Experimental results. Effect of transient current over the switching harmonic impedance. From top to bottom: a) battery voltage, b) battery current, c) $0 \mathrm{~Hz}$ impedance, d) switching harmonic impedance.

experimentally evaluated under a reduced number of operating conditions, being its extension to all the different operating points focus of current research. Using a dc/dc interleaved converter, the module high-frequency impedance has been measured and its matching to a high-frequency model based on frequency fitting has been demonstrated. Based on the developed model, it has been demonstrated that one of the inductors exhibits a nearly linear variation with respect to the SoC. Considering the inductive behavior of the battery model at high-frequency, a discussion about possible injection signals to be used has been carried out. Two alternatives were evaluated for estimating the SoC: 1 ) additional injection signal and 2) use of the switching harmonics. Both alternatives have been tested, being the results for the first switching harmonic more promising due to the reduced impact of the converter filter and the higher sensitivity of the model parameters to the SoC in the switching frequency region.

\section{REFERENCES}

[1] S. Vazquez, S. M. Lukic, E. Galvan, L. G. Franquelo, and J. M. Carrasco, "Energy storage systems for transport and grid applications," IEEE Transactions on Industrial Electronics, vol. 57, no. 12, pp. 3881-3895, Dec 2010.

[2] X. Luo, J. Wang, M. Dooner, and J. Clarke, "Overview of current development in electrical energy storage technologies and the application potential in power system operation," Applied Energy, vol. 137, pp. 511-536, 2015. [Online]. Available: http://www.sciencedirect.com/science/article/pii/S0306261914010290

[3] T. Horiba, "Lithium-ion battery systems," Proceedings of the IEEE, vol. 102, no. 6, pp. 939-950, June 2014.

[4] H. Rahimi-Eichi, U. Ojha, F. Baronti, and M. Y. Chow, "Battery management system: An overview of its application in the smart grid and electric vehicles," IEEE Industrial Electronics Magazine, vol. 7, no. 2, pp. 4-16, June 2013.
[5] L. Lu, X. Han, J. Li, J. Hua, and M. Ouyang, "A review on the key issues for lithium-ion battery management in electric vehicles," Journal of Power Sources, vol. 226, pp. 272-288, 2013. [Online]. Available: http://www.sciencedirect.com/science/article/pii/S0378775312016163

[6] W. Waag, S. Käbitz, and D. U. Sauer, "Experimental investigation of the lithium-ion battery impedance characteristic at various conditions and aging states and its influence on the application," Applied Energy, vol. 102, pp. 885-897, 2013, special Issue on Advances in sustainable biofuel production and use - $\{\mathrm{XIX}\}$ International Symposium on Alcohol Fuels - \{ISAF\}. [Online]. Available: http://www.sciencedirect.com/science/article/pii/S030626191200671X

[7] B. Schweighofer, K. M. Raab, and G. Brasseur, "Modeling of high power automotive batteries by the use of an automated test system," IEEE Transactions on Instrumentation and Measurement, vol. 52, no. 4, pp. 1087-1091, Aug 2003.

[8] M. Charkhgard and M. Farrokhi, "State-of-charge estimation for lithiumion batteries using neural networks and ekf," IEEE Transactions on Industrial Electronics, vol. 57, no. 12, pp. 4178-4187, Dec 2010.

[9] A. Fotouhi, D. J. Auger, K. Propp, S. Longo, and M. Wild, "A review on electric vehicle battery modelling: From lithiumion toward lithium-sulphur," Renewable and Sustainable Energy Reviews, vol. 56, pp. 1008-1021, 2016. [Online]. Available: http://www.sciencedirect.com/science/article/pii/S1364032115013921

[10] A. Bizeray, S. Duncan, and D. Howey, "Advanced battery management systems using fast electrochemical modelling," in IET Hybrid and Electric Vehicles Conference 2013 (HEVC 2013), Nov 2013, pp. 1-6.

[11] J. Chiasson and B. Vairamohan, "Estimating the state of charge of a battery," IEEE Transactions on Control Systems Technology, vol. 13, no. 3, pp. 465-470, May 2005.

[12] X. Lin, "Theoretical analysis of battery soc estimation errors under sensor bias and variance," IEEE Transactions on Industrial Electronics, vol. PP, no. 99, pp. 1-1, 2018.

[13] D. Andre, C. Appel, T. Soczka-Guth, and D. U. Sauer, "Advanced mathematical methods of soc and soh estimation for lithium-ion batteries," Journal of Power Sources, vol. 224, pp. 20-27, 2013. [Online]. Available: http://www.sciencedirect.com/science/article/pii/S0378775312015303

[14] W. He, N. Williard, C. Chen, and M. Pecht, "State of charge estimation for li-ion batteries using neural network modeling and unscented kalman filter-based error cancellation," International Journal of Electrical Power \& Energy Systems, vol. 62, pp. 783-791, 2014. [Online]. Available: http://www.sciencedirect.com/science/article/pii/S0142061514002646

[15] H. He, R. Xiong, X. Zhang, F. Sun, and J. Fan, "State-of-charge estimation of the lithium-ion battery using an adaptive extended kalman filter based on an improved thevenin model," IEEE Transactions on Vehicular Technology, vol. 60, no. 4, pp. 1461-1469, May 2011.

[16] S. De Breucker, "Impact of dc-dc converters on li-ion batteries," Ph.D. dissertation, Katholieke Universiteit Leuven, 12-Dec-2012.

[17] P. García, S. Saeed, Á. Navarro-Rodríguez, J. Garcia, and H. Schneider, "Switching frequency optimization for a solid state transformer with energy storage capabilities," in 2016 IEEE Energy Conversion Congress and Exposition (ECCE), Sept 2016, pp. 1-8.

[18] B. Gustavsen and A. Semlyen, "Rational approximation of frequency domain responses by vector fitting," Power Delivery, IEEE Transactions on, vol. 14, no. 3, pp. 1052-1061, 1999.

[19] Y. Zhang, C.-Y. Wang, and X. Tang, "Cycling degradation of an automotive lifepo 4 lithium-ion battery," Journal of Power Sources, vol. 196, no. 3, pp. 1513-1520, 2011.

[20] D. Howey, V. Yufit, P. D. Mitcheson, G. Offer, N. P. Brandon et al., "Impedance measurement for advanced battery management systems," in Electric Vehicle Symposium and Exhibition (EVS27), 2013 World. IEEE, 2013, pp. 1-7.

[21] J. Wang, K. Zou, C. Chen, and L. Chen, "A high frequency battery model for current ripple analysis," in Applied Power Electronics Conference and Exposition (APEC), 2010 Twenty-Fifth Annual IEEE. IEEE, 2010, pp. 676-680. 